\title{
Expedited method of testing and refinement of refrigeration centrifugal compressors
}

\author{
Igor Sukhomlinov ${ }^{1}$, and Mihail Golovin ${ }^{1, *}$ \\ 1JSC "VNIIKHOLODMASH", Altufevskoe highway 79a-3, Moscow, 127410 Russia
}

\begin{abstract}
Currently existing regulatory documents assume that refrigeration compressors are tested as part of test rigs with heat-exchange equipment. Given the high energy intensity of centrifugal compressors, the drive power of which in cooling mode with a cold capacity of up to $8.0 \mathrm{MW}$ can be 2.0 or more $\mathrm{MW}$, the creation of test rigs for preliminary and acceptance tests at the manufacturer's factory requires a large investment. An alternative to the considered refrigeration centrifugal compressors test method is a test on a test rig in which the agent is partially condensed. In this case, an evaporator and a full-sized condenser are not required. The mathematical model of the refrigeration centrifugal compressors developed by JSC VNIIKHOLODMASH, allows testing of the refrigeration centrifugal compressors in model conditions, when the entire compressor operation cycle at the test rig is carried out in the area of superheated steam. Since the refrigeration centrifugal compressors mathematical model is based on physical simulation of compression processes in model stages at different values of conditional Mach numbers, dimensionless characteristics of model stages can be used to calculate the characteristic of the created compressor when operating under new conditions. The implementation of the model test method provides reduction of testing time due to absence of phase changes of the agent, improves the accuracy of the obtained results, reduces of power consumption, simplifies the test rig, and reduces consumption of the agent for filling the rig and water for cooling the agent.
\end{abstract}

\section{Introduction}

Centrifugal compressors play an important role in the economies of industrialized countries. They are used to compress gases in the chemical, oil, gas and machinebuilding industries, in transport and metallurgy, construction, production of ammonia fertilizers, plastics, petrochemical products, in oil and gas production, in the refrigeration industry, as turbochargers, in gas turbines, in pneumatic plants. The drive of centrifugal compressors accounts for a significant share of all energy consumed. Therefore, the problem of optimal design of centrifugal compressors is very important. Russian and foreign organizations are currently engaged in the researches and improvement of centrifugal compressors [1-9]. Traditional approaches to the design of centrifugal compressors are based on the results of stage models tests that are carried out on test rigs.

\section{Methods}

Currently existing regulatory documents, including the European standard DIN EN 13771-1: 2016 [1], on the basis of which Rossoyuzholodprom has prepared the draft GOST R EN 13771-1-XXXX, assume that refrigeration compressors are tested as part of test rigs with heat-exchange equipment (HEE) that ensures the implementation of the thermodynamic cycle of the chiller, which means that in practice the compressor is within the chiller. Despite the fact that the standard [10] was developed with respect to volume compressors of small and medium refrigeration capacities, the test methods set out in it are also applicable for testing refrigeration centrifugal compressors (RCC). However, given the high energy intensity of centrifugal compressors, the drive power of which in cooling mode with a cold capacity of up to $8,0 \mathrm{MW}$ can be 2,0 or more MW, the creation of test rigs for preliminary and acceptance tests at the manufacturer's factory requires a large investment and will call for high maintenance costs.

The presence of heat exchange equipment, in which the phase change of the agent occurs, leads to a large inertia of the system and, consequently, to an increase in the test time. In addition, as practice shows, in this case it is not possible to ensure sufficient accuracy of the test results.

Of great difficulty is the testing of RCCs designed to work in technological installations, for example, for separation of associated gas, when the heat exchange

\footnotetext{
* Corresponding author: golovinnmv@yandex.ru
} 
equipment is part of the technological installation itself. In this case, it is not possible to obtain compressor characteristics [11].

An alternative to the considered RCC test method is a test on a test rig in which the agent is partially condensed [12]. In this case, an evaporator and a full-sized condenser are not required, since the compression process in the compressor takes place completely in the region of superheated steam, and only part of the agent is condensed, which is necessary to ensure the conditions at the compressor inlet that correspond to the operating conditions as part of the refrigeration machine.

The scheme of the rig and the operation cycle are shown in Figures 1 and 2. The rig is a sealed steam ring, in which a compressor (RCC), a heat exchanger (h.exch), a throttle valve (thr.v), a separator (sp) with a thermostatic valve (trv) are sequentially included to maintain the set temperature at the suction to the compressor. The suction and condensing working pressures are determined by the amount of chargeable refrigerant and the water part of the rig.

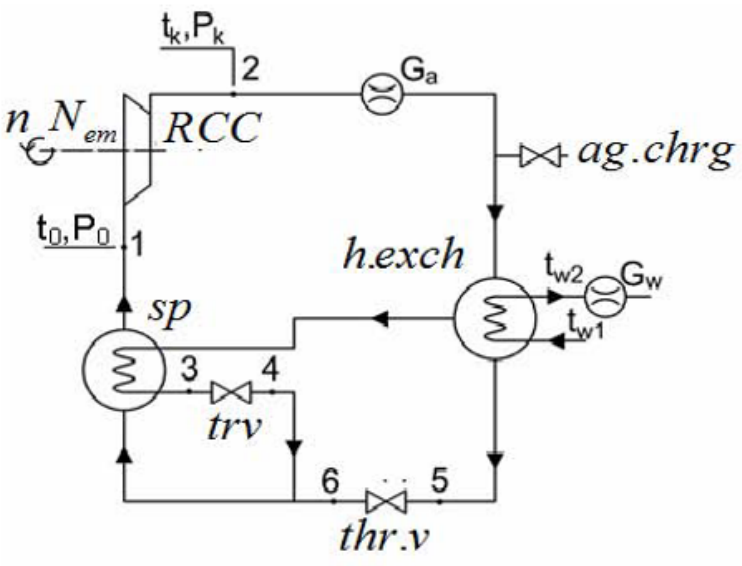

Fig.1. Scheme of the test rig

1 - compressor inlet; 2 - compressor exit; 3 - heat exchanger exit, where $t_{w 1}, t_{w 2}$ is cooling water temperature, $n$ is RCC $\mathrm{RPM}, \mathrm{N}_{\mathrm{em}}$ is electric motor power, $p$ is pressure, $G$ is mass flow rate; $h$ is enthalpy

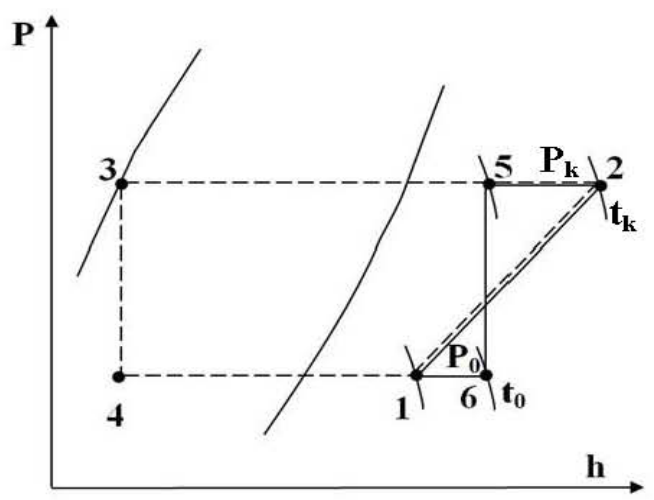

Fig.2. The cycle of processes when testing a compressor

High-pressure refrigerant from the compressor discharge pipe enters the heat exchanger, where it is cooled (process 2-5 in Figure 2) due to cooling water, which is supplied by the pumps of the rig system. Most of the refrigerant is throttled (process 5-6) to the pressure in the separator.

Another part of the refrigerant is cooled and condensed (process 5-3), throttled in the expansion valve to the pressure in the separator (process 3-4) and fed to the cooling of the main refrigerant stream to a predetermined temperature at the suction in the compressor (processes 4-1 and 6-1).

This approach can significantly reduce the cost of the rig, but the presence of an agent in the liquid phase leads to a high inertia of the system, significantly increasing the test time, which is especially important if you need to perform final adjustments to the compressor's flow path.

\section{Results}

The mathematical model (MM) of the RCC developed by JSC VNIIKHOLODMASH for selecting the operating and geometrical parameters of the compressor [13], which proved its reliability in organizing serial production at the Kazancompressormash JSC of the third generation of refrigeration machines of the TXMV type, allows testing of the RCC in model conditions, when the entire compressor operation cycle at the test rig is carried out in the area of superheated steam.

To implement this cycle of operation, the conditions at the inlet to the compressor must be changed in the direction of decreasing pressure and increasing temperature. In this case, the agent's properties will inevitably change, such as the volumetric index of the compression isentropic $\mathrm{kv}$, as well as the relative Mach number, which, based on the theory of similarity of compression processes in RCC [14], are the main ones when maintaining geometric similarity and selfsimilarity of flow regimes that determine dimensionless characteristics stages, and, consequently, the compressor. However, for the RCC test conditions used in chillers operating on R134a agent, the influence of these values, as shown in [12], is not large, amounting to approximately $1,5 \%$, i.e. significantly less than the respective experimental error. In addition, taking into account that the influence of the kv index decreases with a decrease in the relative Mach number, its decrease at a constant rotor speed compensates for the negative effect of the $\mathrm{kv}$ index on the dimensionless characteristics of the stages.

Since the RCC MM is based on physical simulation of compression processes in model stages at different values of conditional Mach numbers [15], dimensionless characteristics of model stages can be used to calculate the characteristic of the created compressor when operating under new conditions. In this case, confirmation of the design dimensionless performance of the compressor tested under model conditions will be sufficient to confirm that the compressor meets the specification. Transition to compressor dimensional characteristics is performed through thermodynamic cycle parameters as per RCC MM. At the same time, the type of cycle - simple or double-throttled agent cycle does not matter, as the tests confirm the validity of the accepted dimensionless characteristics of the stages, 
which are used in determining the compressor characteristic, both in model and in-kind operating conditions. Therefore, if the RCC is designed to operate in a double throttling cycle of the agent, the compressor characteristic under model operating conditions is calculated without regard to the intermediate steam supply of the agent.

The diagram of the test rig of the RCC under model conditions is shown in Figure 3 (the legend is identical to that of Figure 1), and the compression processes in the RCC for the operating conditions as part of the RM and model conditions are shown in Figure 4.

The possibility of using the model test method for RCC, the stage parameters of which are selected without the use of RCC MM $[15,16]$ can be interesting. This task has been described by testing a two-stage compressor for operation in water cooling machines on an agent R134a in boiling and condensation mode $\mathrm{t}_{0} / \mathrm{t}_{\mathrm{k}}=$ $+3 /+40^{\circ} \mathrm{C}$. Two versions of compressor flow path (FP) are considered - one on the basis of stages with vaneless diffuser (VLD), the other on the basis of stages with combined diffuser (VLD-VD).

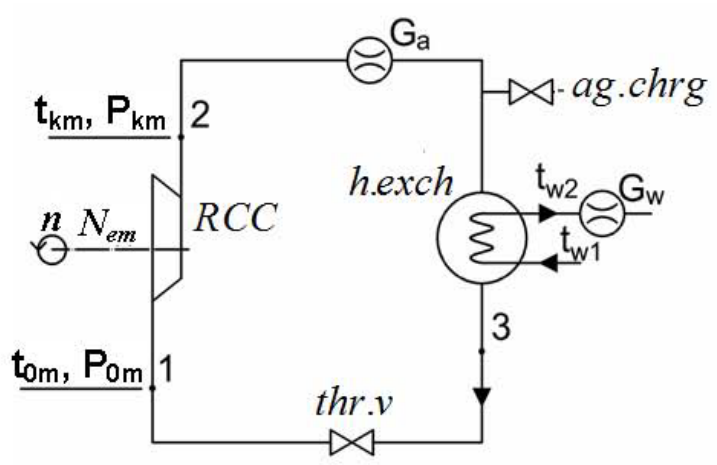

Fig. 3. Scheme of the test rig for model tests

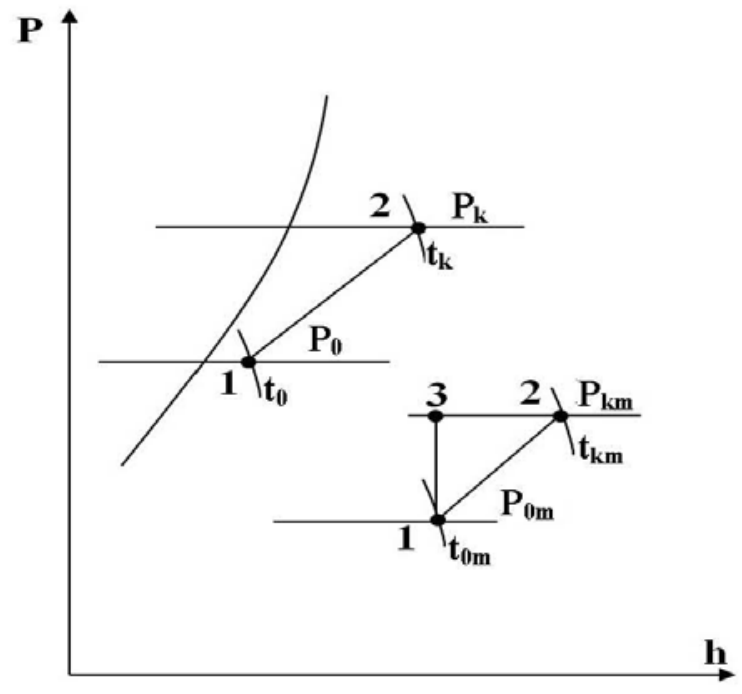

Fig.4. The cycle of processes when testing a compressor, where

$\mathrm{t}_{0}, \mathrm{P}_{0}, \mathrm{t}_{\mathrm{k}}, \mathrm{P}_{\mathrm{k}}$ is temperature and pressure at inlet and outlet to RCC in RM cycle; $\mathrm{t}_{0 \mathrm{~m}}, \mathrm{P}_{0 \mathrm{~m}}, \mathrm{t}_{\mathrm{km}}, \mathrm{P}_{\mathrm{km}}$ is the same in model conditions.
The test was carried out by comparing the compressor characteristics calculated from the RCC MM using the dimensionless characteristics of the stages from the bank and the compressor characteristic calculated from the dimensionless compressor characteristics obtained under model conditions.

Figures 5 and 6 show the dimensionless characteristics of a compressor having a flow path with VLD as a dependence of isentropic efficiency $\eta_{\mathrm{s}}$ and head coefficient $\Psi_{\mathrm{s}}$ of flow rate coefficient $\Phi_{0}$.

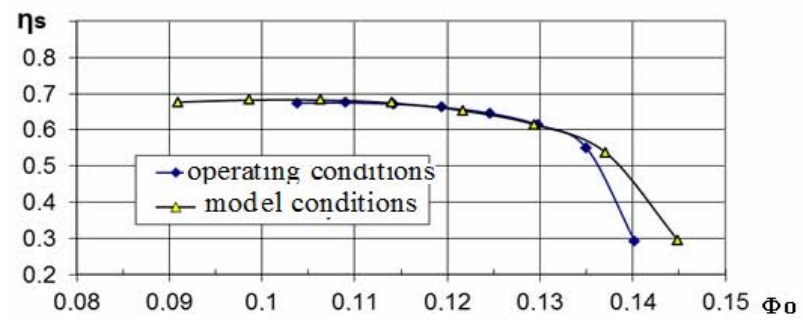

Fig.5. Compressor efficiency characteristic (VLD flow path).

As can be seen from the results, the main difference in dimensionless characteristics is obtained in the area of high flow rate. In addition, due to the mismatch of operation of the stages during operation under model conditions, the zone of stable operation of the compressor has significantly increased towards lower productivity. The difference in dimensional characteristics is slightly larger, but the error in determining performance in the calculated mode is less than $3 \%$.

A similar result was obtained for the compressor with the combined diffuser. However, the difference in dimensionless characteristics in the area of high flow rates due to the influence of the vane part of the diffusers has proved to be large. The performance measurement difference was also less than $3 \%$.

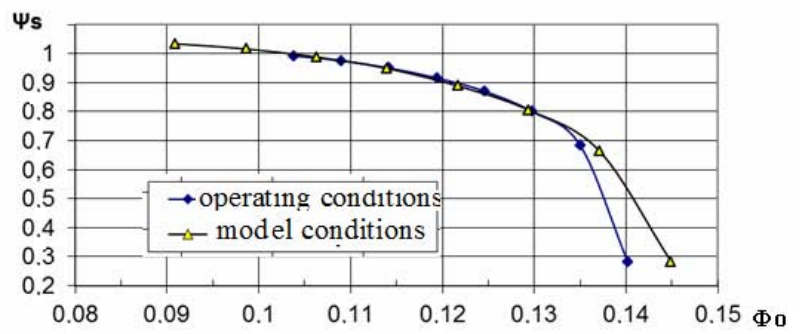

Fig.6. Compressor head coefficient characteristic (VLD flow path).

Therefore, such method of express assessment of compliance of compressor parameters to technical specification and for determination of necessity of finishing works can be used with further refinement of surge boundary on the test rig with partial condensation.

Figures 7 and 8 show the compressor characteristics as a change in the effective power and cooling capacity from the flow rate and operation mode for the condensate temperature $t_{k}$ at boiling point $t_{0}=+3^{\circ} \mathrm{C}$. 


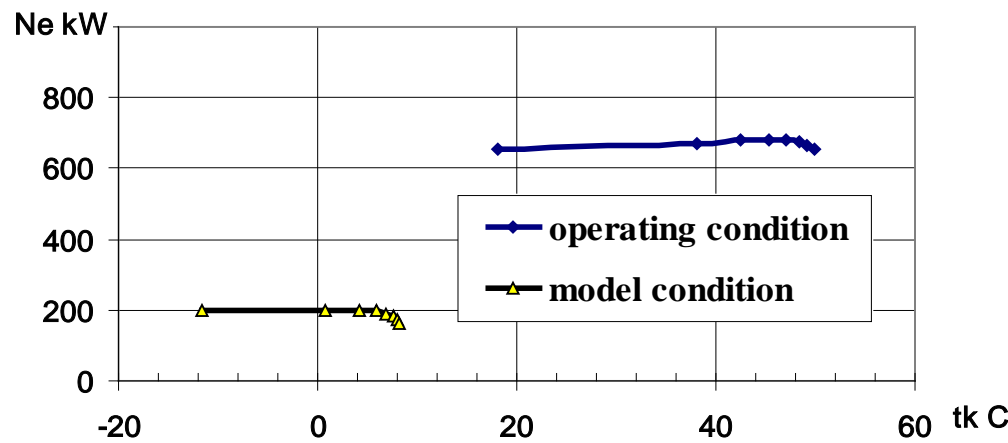

Fig.7. Power consumption of the compressor (VLD-VD flow path).

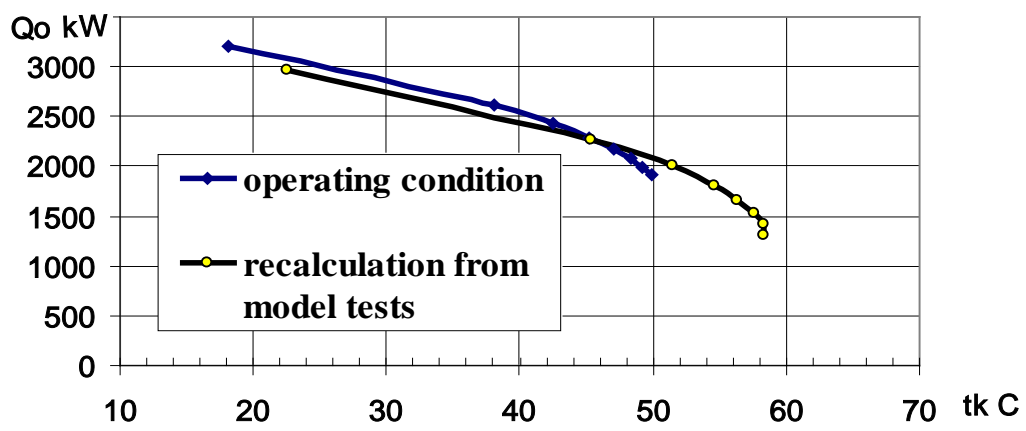

Fig.8. The cooling capacity of the compressor (VLD-VD flow path).

\section{Conclusion}

Thus, the implementation of the model test method provides the following advantages:

- two to three times reduction of testing time due to absence of phase changes of the agent;

- improvement of the accuracy of the obtained results due to the possibility of measuring flow rate not through thermal balances, but directly by means of any measuring technology;

- reduction of power consumption, for example, in the considered cases more than 3 times, due to reduction of mass flow rate by reduction of pressure at the compressor inlet from 3 to 1 atmosphere;

- simplicity of the test rig and, therefore, its lower cost;

- lower consumption of the agent for filling the rig and water for cooling the agent.

\section{References}

1. G.N. Den, Design of the flow path of centrifugal compressors,230 (1980)

2. I.G. Khisameev, V.A. Maximov, G.S. Batkis and Y.Z. Guzelbaev, Design and operation of industrial centrifugal compressors, 671 (2012)

3. Y. Galerkin, A. Rekstin and A. Drozdov International Conference on Compressors and their Systems (2017)

4. L. Marenina, Y. Galerkin and K. Soldatova, Int. Journ. of Mech. Engin. and Rob. Res., 7 (6), 656661 (2017)
5. A.F. Rekstin, A.A. Drozdov, O.A. Solovyeva and Y.B. Galerkin, AIP Conf. Proc. 2007, 030035-1030035-12 (2018)

6. Y. Galerkin, A. Drozdov and O. Solovyeva Proceedings of 13th European Conference on Turbomachinery Fluid dynamics \& Thermodynamics, ETC2019-329, (2019)

7. Yu.B. Galerkin, A.F. Rekstin, K.V. Soldatova and A.A. Drozdov, AIP Conf. Proc., 030018-03001811 (2019)

8. Yu.B. Galerkin, V.B. Semenovskiy and K.V. Soldatova, AIP Conf. Proc. 2141, 030026-030026-8 (2019)

9. D. Japikse, JANNAF Conference (1998).

10. DIN EN 13771-1:2016 Kältemittel-Verdichter und Verflüssigungssätze für die Kälteanwendung Leistungsprüfung und Prüfverfahren - Teil 1: Kältemittel-Verdichter; Deutsche Fassung EN 13771-1 (2016)

11. K.K.Gresh, Hydrocarbon Processing, 77, 1, 55-58. (1998)

12. I. Ya. Sukhomlinov, M.V. Golovin and A.Yu. Znamennikov, Refr. Equip. 12, 28-32 (2018)

13. I.Ya. Sukhomlinov, Refr. Equip., 8, 29-31. (1986)

14. F.M. Chistyakov, Refrigeration turbine units. 288 (1967)

15. I.Ya. Sukhomlinov, Compr. Techn. and pneum., 2. 25-30 (2010)

16. I.Ya. Sukhomlinov, D.L. Slavutsky and M.V. Golovin, Refr. Techn. 9, 10-12 (2000) 